\title{
Isolation, Characterization and Antibacterial Susceptibility test of Acinetobacter species obtained from Tertiary Care Hospital
}

\author{
Preeti Chandra ${ }^{1}$, Vineeta Mittal $^{2}$ and Rachna Chaturvedi ${ }^{1 *}$ \\ ${ }^{1}$ Amity Institute of Biotechnology, Amity University Uttar Pradesh Lucknow, India \\ ${ }^{2}$ Ram Manohar Lohia Institute of Medical Sciences Lucknow, India \\ *Corresponding author
}

\begin{tabular}{|l|}
\hline Ke y w o r d s \\
Acinetobacter \\
species, Tertiary care \\
hospital, Nosocomial \\
infection, Antibiotic \\
susceptibility, \\
Biochemical \\
identification, Disc \\
diffusion method.
\end{tabular}

\section{Introduction}

Acinetobacter species has arisen as significant and challenging human pathogen as it is the causal agent of numerous kinds of infections in humans like nosocomial pneumonia, which is most often related to endotracheal tubes or tracheostomies, endocarditis, meningitis, septicaemia, skin, wound infections, UTI and bacteraemia peritonitis in patients receiving peritoneal dialysis (Winn et al., 2006; Oberoi et al., 2009). Acinetobacter sometimes "colonize" or live in a patient without producing infection or symptoms, especially in tracheostomy sites or exposed wounds. Presently, A. baumannii is becoming an imperative developing nosocomial pathogen universal and is liable for $2-10 \%$ of all the 
Gram-negative infections (Richet et al., 2006). It is characterized as second most offending one after Pseudomonas aeruginosa amongst the nosocomial, aerobic, nonfermentative, gram negative bacilli pathogens. Acinetobacter species are saprophytic, ubiquitous and an important nosocomial pathogen due to its ability for persistence in the hospital environment on a wide range of dry and moist areas (Mindolli et al., 2010). The genus Acinetobacter is now defined as Gram-negative non-fermenting bacilli, strictly aerobic, non-motile, catalase positive, and oxidase negative. Acinetobacter species generally form smooth and sometimes mucoid colonies on solid media, with a colour ranging from white to pale yellow or greyish-white. It has been isolated from the samples like human sputum, blood, urine and pus in patients admitted in hospital (Baltimore et al., 1989 and Rosenthal et al., 1975).

Despite of the increasing significance and frequency of antibiotic resistant activity of Acinetobacter, numerous clinicians still lacking an appreciation of the potential importance of these organisms in hospitals. Since its first detection, A. baumannii has become resistant to numerous common antibiotics due to mutually intrinsic mechanisms and its ability to acquire drug resistance determinants. The accumulative occurrence of multi-drug and pan-drug resistant $A$. baumannii strains found in clinics has extracted it one of the few important nosocomial pathogens, only next to Pseudomonas aeruginosa amongst nonfermentative gram-negative bacteria (Dijkshoorn et al., 2007; Navon-Venezia et al., 2005). A. baumannii is resistant to dehydration, UV radiation, communal chemical sanitizers, and detergents, making it extremely challenging task to destroy $A$. baumannii contaminations from hospital surroundings, especially catheter-related devices used in intensive care units (ICU).
Antibiotic susceptibility pattern of Acinetobacter could differ extensively in nature and between different units of the same hospital at different time points. It is very much necessary to know about the alteration in Acinetobacter resistogram, for a periodic observation of these pathogens to get suitable group of treatment (Prashanth et al., 2004 and Lone et al., 2009) Due to random antibiotic resistance patterns of clinical strains of Acinetobacter, it is necessary to know the institutional widespread susceptibility profiles of this pathogen. In India a small number of studies on Acinetobacter species have been reported especially in Uttar Pradesh and in analysis of their increasing significance in nosocomial infections, more study is needed in this part. Accordingly, this study is undertaken to isolate the Acinetobacter species from different clinical samples via simplified phenotypic identification procedure and to determine the antibiotic susceptibility and resistance pattern of these isolates.

\section{Materials and Methods}

\section{Processing of sample}

The study was carried out in the Department of Microbiology of Ram Manohar Lohia Institute of Medical Sciences, Lucknow over a period of one year. A total of 1040 clinical samples received in the Department of Microbiology for bacterial culture and sensitivity from indoor and outdoor patients were included in the study.

\section{Isolation of bacteria}

Isolation is done for different samples by using different type of media.Blood agar and MacConkey agar was used for pus and sputum samples. CHROM and CLED media were used for urine sample. Blood culture was done by using automated blood culture system. 


\section{Identification of bacteria}

Primary identification of isolates was based on colonial morphology, and different biochemical characteristics through pattern description in Bergey's manual of determinative bacteriology (Holt et al., 1994 and MacFaddin, 2000). Gram staining was done on isolates. Culture positive isolates were further identified by biochemical tests. Acinetobacter species were differentiated by glucose test.

\section{Antimicrobial susceptibility test}

Antimicrobial susceptibility test for Acinetobacter species was done using most common and therapeutically and commercially important antibiotics by Kirby Bauer disk diffusion method on Mueller Hinton agar allowing to norms of Clinical Laboratory Standards institute (CLSI) CLSI document M100-S17 (Wayne, 2007). The antibiotics used in present study were as Ampicillin sulbactam (As), Cefoperazonesulbactam (Cfs), Ceftriaxone (Ctx), Ceftazidime (Caz), Gentamycin (G), Chloramphenicol (Cmp), Ciprofloxacin (Cip), Imipenem (Imp), Amikacin (Ak), Tetracycline (Tet), Tobramycin (Tob), Doripenem (Dori), Doxycycline (Do), Levofloxacin (Levo).

\section{Results and Discussion}

Out of 1040 samples, 359 (34.5\%) were found to be Gram-negative bacteria. Of total Gramnegative organisms, 176 (49\%) were nonfermenter. Of total non-fermenter organisms, Acinetobacter species was 30 (34\%).Out of total 1040 samples, 30 (2.8\%) infections were found to be due to Acinetobacter. In this study the isolates of Acinetobacter species were isolated from different samples blood samples $10(33.3 \%)$ followed by urine sample 9 (30\%), pus $7(23.3 \%)$, and sputum $4(13.3 \%)$ (Table 1) and different environment, i.e. 15
$(50 \%)$ isolates were from ICU, 9 (30\%) from surgical ward and $6(20 \%)$ from gastro ward (Table 2). This study reveals that Acinetobacter infection were common in patients in age group $>50$ years $13(43.3 \%)$ followed by $>30$ years $17(56.6 \%)$ and in male patients $18(60 \%)$ followed by female patients $12(40 \%)$. In the present study different antibiotics showed resistance and susceptibility against isolates of Acinetobacter (Table 3 and Fig. 1). All the antibiotics have variation in their resistance and susceptibility patterns against isolates obtained from different sources and from different environment (Table 4). Maximum resistance was recorded for Imipenem (76\%), followed by Ceftazidime (70\%), Tobramycin (66.6\%), Chloramphenicol (63\%), Doripenem (60\%) Ciprofloxacin (60 \%) Tetracycline (56.6\%), Ceftriaxone $(56.6 \%)$, Doxycycline 16 (53.6\%), Levofloxacin 15 (50\%), Cefoperazone 13 (43\%), Ampicillin 8 $(26.6 \%)$, Amikacin $(10 \%)$ and Gentamycin $(6.6 \%)$. Less resistance was observed in Gentamycin (Table 3 and Fig. 1).

Of the "newer" pathogens currently identified, Acinetobacter plays an important role in colonization and infection of patients admitted to hospitals. They have been concerned in a variety of nosocomial infections, including bacteraemia, urinary tract infections and secondary meningitis and nosocomial pneumonia, especially ventilator associated pneumonia in patients restricted to intensive care units (ICU's). Such infections are frequently very complicated to treat for the clinician because of extensive resistance of the virulent organism to a hugefigure of antibiotics (Bergone Berezin E and Towner KJ1996). Normally Acinetobacter isolated from normal skin and mucous membranes are reported to cause serious and sometimes fatal infections (Pal RB and Kale VV1981). In present study, Acinetobacter accounted for359 $(34.5 \%)$ of total positive culture, 176 $(49 \%)$ of non-fermenter, $88(50 \%)$ of non- 
motile and $2.8 \%$ of total samples cultures. The present study is supported by previously, published studies which have accounted
12.9\% (Lahiri et al., 2004) and 4.8\% (Lone et al., 2009) of Acinetobacter isolates from total infected samples, respectively.

Table.1 Sources of Acinetobacter species

\begin{tabular}{|l|l|l|}
\hline Samples & No. of Samples & $\%$ of sample \\
\hline Blood & 10 & $33.3 \%$ \\
\hline Urine & 9 & $30 \%$ \\
\hline Pus & 7 & $23.3 \%$ \\
\hline Sputum & 4 & $13.3 \%$ \\
\hline
\end{tabular}

Table.2 Percentage of isolates' environment

\begin{tabular}{|l|l|l|}
\hline Environment & No. of isolates & $\%$ of isolates \\
\hline ICU ward & 15 & $50 \%$ \\
\hline Surgical ward & 9 & $30 \%$ \\
\hline Gastro ward & 6 & $20 \%$ \\
\hline
\end{tabular}

Table.3 Antibiotic resistance pattern of Acinetobacter species

\begin{tabular}{|l|l|l|}
\hline Antibiotics & $\begin{array}{l}\text { No. of Resistance } \\
(\mathrm{n}=30)\end{array}$ & $\%$ of Resistance \\
\hline Imipenem & 23 & $76.6 \%$ \\
\hline Ceftazidime & 21 & $70 \%$ \\
\hline Tobramycin & 20 & $66.6 \%$ \\
\hline Chloramphenicol & 19 & $63.3 \%$ \\
\hline Doripenem & 18 & $60 \%$ \\
\hline Ciprofloxacin & 18 & $60 \%$ \\
\hline Tetracycline & 17 & $56.6 \%$ \\
\hline Ceftriaxone & 17 & $56.6 \%$ \\
\hline Doxycycline & 3 & $53 \%$ \\
\hline Levofloxacin & 16 & $50 \%$ \\
\hline Cefoperazone sulbactam & 13 & $43.3 \%$ \\
\hline Ampicillin sulbactam & 8 & $26.6 \%$ \\
\hline Amikacin & 3 & $10 \%$ \\
\hline Gentamycin & 2 & $6.6 \%$ \\
\hline
\end{tabular}

Table.4 Antibiotics resistances pattern

\begin{tabular}{|l|l|l|}
\hline Source & $\begin{array}{l}\text { No. of Acinetobacter } \\
\text { isolates }(\mathrm{n}=30)\end{array}$ & Resistances pattern \\
\hline Blood & 10 & Imipenem $>$ Tobramycin $>$ Doripenem \\
\hline Urine & 9 & Imipenem $>$ Chloramphenicol $>$ Ceftriaxone \\
\hline Pus & 7 & Ceftazidime $>$ Doripenem $>$ Levofloxacin \\
\hline Sputum & 4 & Imipenem $>$ Doripenem $>$ Tobramycin \\
\hline
\end{tabular}


Fig.1 Antibiotics resistance (\%) for different isolates of Acinetobacter species

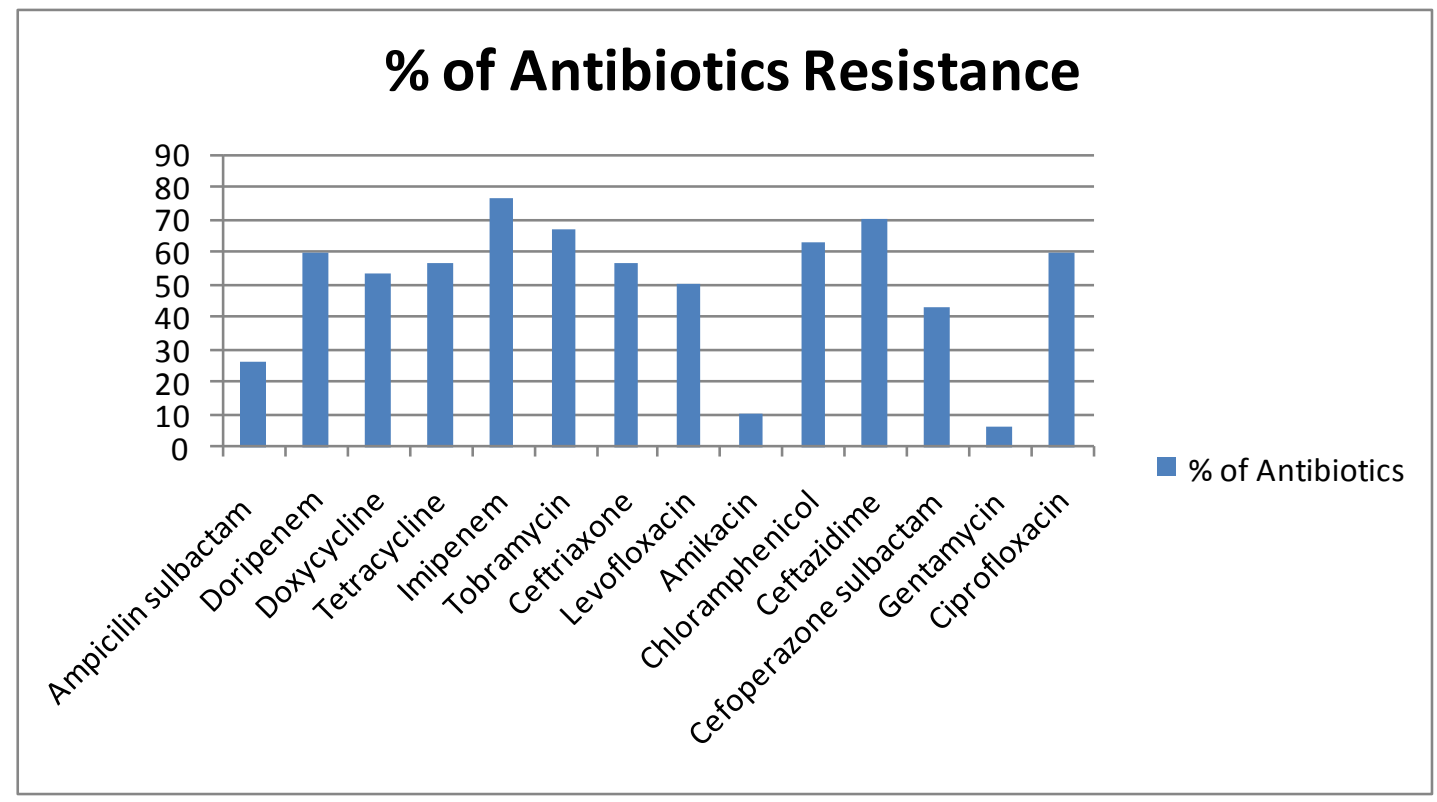

Pseudomonas species was the most common nonfermenter isolated in previous studies (Neetu Gupta et al., 2015). Fontana et al., 2008 found that the A. baumannii was the most common pathogen (7\%) among Gram negative bacteria isolated from patients of intensive care units in France hospitals. While, it has been always considered as less virulent amongst other pathogenic microbes however (Joly-Guillou, 2005), recommend that occasionally it can be extremely pathogenic and cause invasive diseases. The members of this genus are usually inhabitants of human skin, throat, respiratory and intestinal tract of hospitalized patients; additional reservoirs may involve the medical equipment used in the hospital environment besides the patients and hospital staff may also be act as reservoir for this pathogen (Towner, 2006).

This study showed that large number of isolates were isolated from blood samples $(33.3 \%)$, followed by urine (30\%), pus $(23.3 \%)$ and sputum $(13.3 \%)$ while in various countries, studies on Acinetobacter isolation have shown predominance in urine $(21-27 \%)$ and tracheobronchial secretions (24.8-48.8\%)
(Lahiri et al., 2004). According to various studies Acinetobacter were commonly obtained from patients admitted in ICU and who have undergone invasive process or surgery or on advanced generation antimicrobial drugs. In this present study also most of the isolates were obtained from ICU which is related to the research conducted by Mindolli et al., (2010), in present study 15 (50\%) Acinetobacter isolates were obtained from ICU out of them 10 (33.3\%) from blood which is supported with previous reports of Seifert et al., (1995) who observed maximum Acinetobacter isolates were obtained from blood samples and from ICU. Bacteraemia due to Acinetobacter occur most frequently in critically ill patients particularly admitted in ICUs as these patients usually require prolonged hospital stay, need repeated invasive procedures and frequently receive treatment with broad spectrum antimicrobials (Cisneros and Rodríguez-Baño, 2002).

The distribution of Acinetobacter in clinical samples perhaps is due to its capability to cause various nosocomial infections and resistance to a broad variety of antibiotics. Moreover, some authors stated that the source 
of infection maybe from endogenous routes slightly than the exogenous routes by sink, taps and hands of hospital staff (Thomson, 2004). In India Acinetobacter is recorded for about $13.2 \%$ of nosocomial infection in ICU patients (Patwardhan et al., 2008). In our study Acinetobacter infection was more common in patients in age group $>50$ years [13 (43.3\%)] followed by $>30$ years [17 (56.6\%)]. Neeta Gupta et al., (2015) also observed that the infection was common in patients of age group $>50$ years followed by 0-10 years age group. In the study by Mindolli et al., (2010) isolates Acinetobacter were in age group $>45$ years possibly due to weakened immune system and associated chronic diseases in these age groups.

One of the most prominent properties of genus Acinetobacter is the ability to develop antibiotic resistance very hastily in response to challenge through new antibiotics. In this present study, maximum resistance is observed to Imipenem (76\%), Ceftazidime (70\%), Tobramycin (66.6\%), Chloramphenicol 63.3\%), Doripenem (60\%), Ciprofloxacin (60 \%) Ceftriaxone (56.6\%) Levofloxacin $(56.6 \%)$ and less resistance to Ampicillin sulbactam (26.6\%), Amikacin (10\%), Gentamycin (6.6\%), respectively.

Neetu Gupta et al., (2015) observed that Acinetobacter showed maximum resistance to piperacillin $(55 \%)$, followed by Ceftriaxone $(46 \%)$ and Ceftazidime (46\%). However Rahbar et al., (2010) found high rate of resistance to $A$. baumannii for Ceftriaxone (90.9\%), Piperacillin (90.9\%), Ceftazidime (84.1\%), Ciprofloxacin $(90.9 \%)$ which is similar to our study. Rahbar et al., (2010) observed that Imipenem was the most effective antibiotic, which is inconsistent with our observation. In our study Imipenem (76\%) showed highest resistance to Acinetobacter species.
Maximum resistance was observed in ICU isolates in comparison to wards where Acb complex was most prevalent. In ICUs most sensitive drug was ciprofloxacin (69\%) followed by Imipenem (64\%). In the study conducted by Cisneros and Rodríguez-Baño (2002) they found that many isolates of Acinetobacter species were resistant to almost all antibiotics routinely used in the ICUs of their hospital. In the antibiotic sensitivity studies conducted in Pakistan resistance rates of A. baumannii are reported as 32-100\% against ciprofloxacin, $91-100 \%$ against Cefepime, 90-92\% against Piperacillintazobactam, 24-94\% against Amikacin and $18-85 \%$ against Gentamycin (Gozutok et al., 2013). It is in support of our study which showed minimum resistance against Gentamycin and Amikacin. In some previous studies it was found that the resistance rates were 84.9 against Ciprofloxacin, $95.5 \%$ against Cefepime, 89.2\% against Piperacillintazobactam, $86.3 \%$ against amikacin and $76.5 \%$ against Gentamicin and these results will remove these drugs from being an option for treatment. These results were not in support of our results which showed Gentamycin is least resistance (Necati Hakyemez et al., 2013).

In conclusion, A. baumannii is a pathogen with rapid spread and extended resistance to even newer antimicrobial agents. Due to its ability to survive in the hospital environment, A. baumannii has the immense potential to cause nosocomial infectionsand also Acinetobacter is now a day a common threat in hospital acquired infection especially in critically ill patients admitted to intensive care unit. Therefore the main aim of this study was to assess the distribution of Acinetobacter species and to determine the detection rate of this microorganism in comparison to other pathogens in different patients suffering from significant infections Acinetobacter species were found to be resistant to most commonly 
used antibiotics. Therefore, identification and expressive antibiotic sensitivity pattern of Acinetobacter helps in developing antibiotic policy against hospital acquired infections. In the present study it was observed that all isolates were resistant to almost all the antibiotics used in the present study except Amikacin and Gentamycin which are susceptible to the Acinetobacter species showing susceptibility in $28(93.3 \%)$ and 27 $(90 \%)$ patients. Finally it is evident from present study in ICU most sensitive drugs are Gentamycin and Amikacin.

\section{Acknowledgement}

The authors are thankful to the Authorities of Ram Manohar Lohia Institute of Medical Sciences Lucknow, India, for providing necessary facilities to conduct the experiments also thankful to authorities of Amity University Uttar Pradesh for their kind support.

\section{References}

Baltimore, R.S., R.L. Duncan, E.D. Shapiro, and S.C. Edberg. 1989. Epidemiology of pharyngeal colonization of infants with aerobic gram-negative rod bacteria. $J$. Clin. Microbiol., 27: 91-95.

Bergone Berezin, E., Towner, K.J. 1996. Acinetobacter species as Nosocomial Pathogens: Microbiological, Clinical, and Epidemiological Features. Clin. Microbiol. Rev., 9: 148-65.

Cisneros, J.M., Rodríguez-Baño, J. 2002. Nosocomial bacteremia due to Acinetobacter baumannii: Epidemiology, clinical features and treatment. Clin. Microbiol. Infect., 8: 687-93.

Dijkshoorn, L., Nemec, A., Seifert, H. 2007. An increasing threat in hospitals: multidrugresistant Acinetobacter baumannii. Nat. Rev. Microbiol., 5(12): 939-951.

Fontana, C., Favaro, M., Minelli, S., Bossa, M.C., Testore, G.T., Leonardis, F. and
Favalli, C. 2008. Acinetobacter baumannii intensive care unit: A novel system to study clonal relationship among the isolates. BMC Infect. Dis., 8: 8-79.

Gozutok, F., Sariguzel, F., Celik, I., Berk, E., Aydin, B., Guzel, D. 2013. Investigation of Antimicrobial Resistance Rates of Acinetobacter baumannii Strains from Nosocomial Infections. ANKEM Derg., 27(1): 7-12.

Holt, J.G., Krieg, N.R., Sneath, H.A., Stanley, J.T. and Williams, S.T. 1994. Bergeys Manual of Determinative Bacteriology. 9th ed., Baltimore; Wiliams and Wilkins, USA.

Joly-Guillou, M.L. 2005. Clinical impact and pathogenicity of Acinetobacter. Clin. Microbiol. Infect., 11: 868-873.

Lahiri, K.K., Mani, N.S., Purai, S.S. 2004. Acinetobacter species as nosocomial pathogen: Clinical significance and antimicrobial sensitivity. Med. J. Armed Forces India, 60: 7-10.

Lone, R., Shah, A., Kadri, S.M., Lone, S., Shah, F. 2009. Nosocomial multi-drug resistant Acinetobacter infections-clinical findings, risk factors and demographic characteristics. Bangladesh J. Med. Microbiol., 03: 34-8.

MacFaddin, J.F. 2000. Biochemical Tests for Identification of Medical Bacteria (3rd ed.), Lippincott Williams and Wilkins, USA.MacFaddin, J.F. (2000). Biochemical Tests for Identification of Medical Bacteria (3rd ed.), Lippincott Williams and Wilkins, USA.

Mindolli, P.B., Salmani, M.P., G. Vishwanath and A.R. Hanumanthappa. 2010. Identification and Speciation of Acinetobacter and Their Antimicrobial Susceptibility Testing. Al Ameen J. Med. Sci., 3(4): 345-49

Navon-Venezia, S., Ben-Ami, R., Carmeli, Y. 2005. Update on Pseudomonas aeruginosa and Acinetobacter baumannii infections in the healthcare setting. Curr. Opin. Infect. Dis., 18(4): 306-313.

Necati Hakyemez, I., Kucukbayrak, A., Tas, T., BurcuYikilgan, A., Akkaya, A., 
Yasayacak, A., and Akdeniz, H. 2013. Nosocomial Acinetobacter baumannii Infections and Changing Antibiotic Resistance. Pakistan J. Med. Sci., 29(5), 1245-1248.

Neetu Gupta, NageswariGandham, Savita Jadhav and Ravindra Nath Mishra. 2015. Isolation and identification of Acinetobacter species with special reference to antibiotic resistance. J. Nat. Sci. Biol. Med., 6(1): 159-162

Oberoi, A., Aggarwal, A., Lal, M. 2009. A decade of underestimated nosocomial pathogen-Acinetobacter in a tertiary care hospital in Punjab. J.K. Sci., 11: 24-6.

Pal, R.B., Kale, V.V. 1981. Acinetobacter calcoaceticus - An opportunistic pathogen. J. Postgrad. Med., 7: 218-21.

Patwardhan, R.B, Dhakephalkar, P.K, Niphadkar, K.B, Chopade, B.A. 2008. A study on nosocomial pathogens in ICU with special reference to multiresistance Acinetobacter baumannii harbouring multiple plasmids. Indian J. Med. Res., 128: $178-187$.

Prashanth, K., Badrinath, S. 2004. Invitro susceptibility pattern of Acinetobacter species to commonly used cephalosporins, quinolones and amino glycosides. Indian J. Med. Microbiol., 22(2): 97-103.

Rahbar, M., Mehrgan, H., Aliakbari, N.H. 2010. Prevalence of antibiotic resistant Acinetobacter baumannii in a 1000-bed tertiary care hospital in Tehran, Iran. Indian J. Pathol. Microbiol., 53: 290-3.
Richet, H. and Fournier, P.E. 2006. Nosocomial infections caused by Acinetobacter: a major threat worldwide. Infect. Control Hosp. Epidemiol., 27: 645-646.

Rosenthal, S., and I.B. Tager. 1975. Prevalence of gram-negative rods in the normal pharyngeal flora. Ann. Intern. Med., 83: 355-357.

Seifert, H., Strate, A., Pulverer, G. 1995. Nosocomial bacteremia due to Acinetobacter baumannii. Clinical features, epidemiology, and predictors of mortality. Medicine (Baltimore), 74: 3409.

Thomson, N. 2004. The role of prophage-like elementsin the diversity of Salmonella enteric serovars. J. Mol. Biol., 339 (2): 279-300.

Towner, K. 2006. The Genus Acinetobacter. Prokaryotes, 6: 746-758.

Wayne, PA, USA: CLSI; 2007. CLSI document M100-S17. Clinical and Laboratory Standards Institute. Performance Standards for Antimicrobial Susceptibility Testing; Seventeenth Informational Supplement

Winn, W.C., Allen, S.D., Janda, W.M., Koneman, E.W., Procop, G.W., Schreckenberger, P.C. 2006. Taxonomy, biochemical characteristics and clinical significance of medically important nonfermenters. In: Darcy P, Peterson N, editors. Koneman's Colour Atlas and Textbook of Diagnostic Microbiology. 6th ed. Philadelphia: Lippincott Williams and Wilkins; pp. 353-5.

\section{How to cite this article:}

Preeti Chandra, Vineeta Mittal and Rachna Chaturvedi. 2017. Isolation, Characterization and Antibacterial Susceptibility test of Acinetobacter Species Obtained from Tertiary Care Hospital. Int.J.Curr.Microbiol.App.Sci. 6(2): 1279-1286. doi: http://dx.doi.org/10.20546/ijcmas.2017.602.144 